\title{
La Medicación: Talón de Aquiles, también en Atención Primaria
}

La Seguridad del Paciente ocupa en la actualidad un lugar prioritario en el desarrollo de las actividades asistenciales de las instituciones sanitarias a nivel mundial, nacional y regional, las cuales están llevando a cabo importantes iniciativas para disminuir los errores de la práctica clínica ${ }^{1}$.

Hasta hace relativamente poco, todo lo referente a la seguridad del paciente se había centrado en el ámbito hospitalario, pero, desde la publicación del estudio APEAS (Estudio sobre la seguridad de los pacientes en atención primaria de salud ${ }^{2}$ ), conocemos lo que ocurre en nuestro ámbito y en nuestro país y se ha comenzado a trabajar en este sentido ${ }^{3}$.

$Y$ es que, si bien la frecuencia de eventos adversos (EA) provocados por la asistencia en atención primaria es baja, comparándola con su ocurrencia en hospitalización, el gran número de consultas que se realizan en nuestro ámbito (300 millones al año en España, aproximadamente ${ }^{4}$ ) hace que, en términos absolutos, el número de EA sea muy alto, incluyendo no pocos de carácter grave, evitables además en su mayoría.

La etiología de los EA es multicausal, estando comprometidos en su origen factores relacionados con el uso de fármacos, con la comunicación, con la gestión y con los cuidados. De la misma forma que ocurre en la asistencia hospitalaria, es la medicación la causa más importante de los incidentes ocurridos, estando implicada en el $48 \%$ de los eventos adversos en Atención Primaria.

Siendo la elaboración de estrategias preventivas de Ios EA en Atención Primaria altamente efectiva, al ser evitables el $70 \%$ de los EA en general y el $80 \%$ de los EA graves en particular, parece entonces, no sólo razonable, sino también necesario ayudar a los profesionales de atención primaria y establecer recomendaciones sobre la mejora de la formación en el adecuado manejo de los medicamentos, estandarizar la presentación de la información y realizar mejoras en la comunicación e información, abordando de esta forma varios de los problemas existentes.

Por todo ello, la planificación de distintas actuaciones de nuestra Gerencia en este sentido se ha visto así respaldada desde el Sistema Nacional de Salud, habiendo sido fundamental el impulso dado por el Servicio de Salud de CastillaLa Mancha (SESCAM), tanto con la publicación del Plan Estratégico de Seguridad del Paciente 20092012, que contempla entre sus líneas estratégicas la prevención de errores de medicación ${ }^{5}$, como con las actuaciones llevadas a cabo durante este periodo de tiempo, prestando especial atención al control de los riesgos relacionados con la prescripción de medicamentos desde nuestras consultas (detección de interacciones farmacológicas, del riesgo de los medicamentos en caso de embarazo y de las alergias a medicamentos ${ }^{6}$ ).

Dadas las consecuencias clínicas que un error de medicación puede tener en el caso de urgencia o emergencia, éstas son consideradas como puntos importantes sobre los que actuar a la hora de evitar errores.

Un gran número de situaciones de emergencia vital y de parada cardiorrespiratoria tienen lugar en atención primaria, siendo la rapidez y la eficacia en la prestación de la asistencia sanitaria vitales en la obtención de resultados favorables.

En la cadena de supervivencia, refiriéndose a la secuencia de actuaciones encaminadas a disminuir la mortalidad en las situaciones de compromiso vital, intervienen diversos factores como la formación y las habilidades de los profesionales ante este tipo de situaciones, el lugar donde se produzca el evento, la disponibilidad de los recursos materiales y humanos (profesionales, material necesario y en perfectas condiciones), así como la ubicación del carro de parada ${ }^{7}$.

Es en estos momentos la utilización de determinada medicación puede considerarse como de alto riesgo, debido a que las consecuencias de un error en su uso durante la actividad asistencial urgente pueden tener como desenlace un daño grave para los pacientes.

Por ello, pensamos que el hecho de tener un sistema de comunicación protocolizada en situaciones de urgencia y disponer de unos criterios mínimos unificados en cuanto a dotación y revisión del material integrante del carro de parada, que permita a los profesionales disminuir la variabilidad de actuaciones, así como responder adecuadamente a la situación con independencia del centro de salud (CS) donde presten sus servicios en ese momento, es trascendente para la seguridad tanto del paciente como del profesional.

Y así, fueron elaborados los dos proyectos que someramente describimos y que creemos es útil difundir: uno consistió en la elaboración de un "Protocolo sobre órdenes verbales en la administración de medicamentos" y el siguiente fue 
dirigido a eludir errores de medicación ocasionados durante el manejo de fármacos de los carros de parada, con la implantación de un "Protocolo de revisión y mantenimiento sistematizado de los medicamentos del carro de parada".

Con el primero de ellos se pretende normalizar la transmisión de la información a la hora de administrar medicamentos, cuando no sea posible dar las órdenes por escrito, con la finalidad de minimizar errores en la transmisión de la información. Este documento fue consensuado por los profesionales de la Comisión del Uso Racional de Medicamentos (CURM) y de la Unidad Funcional de Gestión de Riesgos Clínicos (UFGR). En su elaboración han intervenido farmacéuticos, médicos y enfermeros de atención primaria y hospitalaria, así como especialistas en urgencias y emergencias. Además se ha elaborado un vídeo ilustrativo del citado protocolo, que apoya la difusión del mismo (disponible en: http://gaptalavera.sescam.jccm. es/web1/gaptalavera/prof_calidad/SESCAM_ Ordenes_Verbales.wmv).

El segundo proyecto se llevó a cabo siendo conocedores de que el momento de emergencia en el que se precisa el carro de parada puede ser un período crítico para la comisión de errores a la hora de la administración de los medicamentos. Se trata de facilitar el hecho de revisar, identificar, homogeneizar y realizar una correcta conservación dela medicación delos carros de parada de los puntos de atención continuada (PAC) del área. Para ello, se estableció un listado de medicamentos básicos para el carro de parada, basado en la evidencia disponible, de los PAC de atención primaria y tras auditoría, que puso de manifiesto la gran variabilidad existente en cuanto a medicamentos disponibles en cada carro, localización de los mismos y estado de conservación. Se propuso homogeneizar la medicación de los carros de parada y proceder a la elección de un maletín de medicación para los mismos que permitiera separar adecuadamente y mantener organizados los distintos medicamentos y que fuera manejable y fácilmente identificable por todos los profesionales. Fue sistematizada su revisión mediante un protocolo, se elaboró una tarjeta tamaño bolsillo, fácil de llevar en la bata de nuestros sanitarios en caso de necesidad, donde se recuerdan las pautas posológicas de la medicación más usada en urgencias y, por último, hemos dotado a los CS de los maletines para la medicación de los carros de parada (disponible en: http://gaptalavera. sescam.jccm.es/web1/gaptalavera/prof_farmacia/ Normas_funcionamiento_botiquines.pdf).

Ambos proyectos han sido difundidos a través de sesiones farmacoterapéuticas en todos los CS, dirigidas tanto a médicos como a enfermeros, y hemos podido observar el grado de cumplimiento del protocolo a los 6 meses de la implantación. Es cierto que aún hay margen para la mejora, pero casi un $60 \%$ de los ítems relacionados con su mantenimiento han sido cumplidos.

Son herramientas elaboradas por y para los profesionales sanitarios, con el fin de incrementar la seguridad del paciente en la asistencia sanitaria. Desde la Unidad Funcional de Gestión de Riesgos de la Gerencia de Atención Primaria de Talavera de la Reina insistiremos en su difusión, intentando, además, concienciar de la trascendencia que suponen la vigilancia y la protocolización que se han de seguir cuando se administran medicamentos en atención primaria, incrementando también de esta forma la cultura de seguridad entre nuestros profesionales.

\section{BIBLIOGRAFÍA}

1. Patient safety solutions preamble- May 2007 [Internet] Geneva: World Health Organization; 2007 [acceso 12/08/2011] Disponible en: http://www.ccforpatientsafety. org/common/pdfs/fpdf/presskit/Preamble.pdf

2. Estudio APEAS. Estudio sobre la seguridad de los pacientes en atención primaria de salud [Internet]. Madrid: Ministerio de Sanidad y Consumo; 2008 [acceso 12/08/2011]. Disponible en: http://www.msc.es/organizacion/sns/ planCalidadSNS/resumenEstudioApeas2008.htm.

3. ¿Porqué un monográfico sobre seguridad del paciente en Atención Primaria? Grupo de Seguridad del Paciente de la Sociedad Española de Medicina Familiar y Comunitaria. Revista Clínica Electrónica en Atención Primaria. 2010; Núm. 18. Disponible en: http://ddd.uab.cat/pub/ rceap/18874215n18/18874215n18.html.

4. Actividad asistencial atención primaria sistema nacional de salud. Informe resumen 2007-2008 [Internet]. Madrid: Ministerio de Sanidad, Política Social e Igualdad; [acceso 12/08/2011]. Disponible en: http://www.msps. es/estadEstudios/estadisticas/docs/siap/Informe de Actividad_AP_2007-2008.pdf.

5. Plan Estratégico de Seguridad del Paciente 2009-2012 [Internet]. Toledo: SESCAM; 2008 [acceso 12/08/2011]. Disponible en: http://sescam.jccm.es/web1/ciudadanos/ elSescam/Plan_Estrategico_Seguridad_SESCAM_09-12. pdf.

6. Martín AM. Mejora de la seguridad en la prescripción de medicamentos. Resultados en pacientes del control de interacciones medicamentosas, alegias y del riesgo en embarazo. Boletín Farmacoterapéutico de Castilla La Mancha 2011; 12 (3). Disponible en: http://sescam.jccm. es/web1/profesionales/farmacia/usoRacional/documentos/ fich_descarga_XII_03_Mej_Seg_Pres_Med.pdf.

7. Prácticas para mejorar la seguridad de medicamentos de alto riesgo [Internet]. Madrid: Ministerio de Sanidad y Consumo; 2007. [acceso 12/08/2011]. Disponible en: http://www.ismp-espana.org/ficheros/Practicas $\% 20$ para $\% 20$ mejorar $\% 20$ la $\% 20$ seguridad $\% 20$ de $\% 20 l o s \% 20$ medicamentos\%20de\%20alto\%20riesgo..pdf.

María Belén de la Hija Díaz

Farmacéutica y miembro de la Unidad Funcional de Gestión de Riesgos Clínicos de la Gerencia de Atención Primaria de Talavera de la Reina.

María Luisa Torijano Casalengua Responsable de la Oficina de Calidad y Atención al usuario y miembro de la Unidad Funcional de Gestión de Riesgos Clínicos de la Gerencia de Atención Primaria de Talavera de la Reina. 